\title{
Directional bioconversion and optimization of stevioside into rubusoside by Lelliottia sp. LST-1
}

\author{
Jingyu Huang ${ }^{1}$, Ke Huang ${ }^{1}$, Jing Lin ${ }^{1}$, Hongwei Li ${ }^{1}$, Jiaqi Xin ${ }^{1}$, Junhao Sang ${ }^{1}$, zhiyun \\ Hong ${ }^{1}$, Ziyang $\mathrm{Ma}^{1}$, Guohong Zeng ${ }^{1}$, xiufang $\mathrm{Hu}^{1}$, and $\mathrm{Ou} \mathrm{Li}^{1}$ \\ ${ }^{1}$ Zhejiang Sci-Tech University
}

November 17, 2020

\begin{abstract}
Steviol glycosides, mostly extracted from Stevia rebaudiana Bertoni, are non-caloric, safe natural sweeteners. Steviosides make up more than $50 \%$ of total steviol glycosides but possess a bitter aftertaste, which limits their application as food additives. Trace amounts of rubusosides are acceptable sweeteners, but their supply is limited. In this study, we screened an endophytic bacteria (Lelliottia LST-1) that can specifically convert stevioside into rubusoside and reach a conversion rate of $75.4 \%$ after response surface optimization. Potential $\beta$-glucosidases GH3-1, GH3-2, GH3-3, and GH3-4 based on whole genome sequencing were expressed and interacted with steviol glycosides to explore principle and process of biological transformation. Highperformance liquid chromatography revealed that all enzymes hydrolyzed stevioside and generated rubusoside, but substrate specificity analysis indicated that GH3-2 is the most suitable choice for the durative, steady, and high-efficiency production of rubusoside
\end{abstract}

\section{Directional bioconversion and optimization of stevioside intorubusoside by Lelliottiasp. LST-1}

Jingyu Huang ${ }^{1}$, Ke Huang ${ }^{1}$, Jing Lin ${ }^{1}$, Hongwei Li $^{1}$, Jiaqi Xin ${ }^{1}$, Junhao Sang ${ }^{1}$, Zhiyun Hong ${ }^{1}$, Ziyang Ma ${ }^{1}$, Guohong Zeng ${ }^{1}$, Xiufang $\mathrm{Hu}^{1}$, Ou Li ${ }^{1 *}$

${ }^{1}$ Zhejiang Province key Laboratory of Plant secondary Metabolism regulation, Zhejiang Sci-tech university, Zhejiang 310018, China

*Corresponding author: Ou Li

Tel: 86-13282813228; E-mail:ouli@zstu.edu.cn.com

Abstract

Steviol glycosides, mostly extracted fromStevia rebaudiana Bertoni, are non-caloric, safe natural sweeteners. Steviosides make up more than $50 \%$ of total steviol glycosides but possess a bitter aftertaste, which limits their application as food additives. Trace amounts of rubusosides are acceptable sweeteners, but their supply is limited. In this study, we screened an endophytic bacteria (Lelliottia LST-1) that can specifically convert stevioside into rubusoside and reach a conversion rate of $75.4 \%$ after response surface optimization. Potential $\beta$-glucosidases GH3-1, GH3-2, GH3-3, and GH3-4 based on whole genome sequencing were expressed and interacted with steviol glycosides to explore principle and process of biological transformation. High-performance liquid chromatography revealed that all enzymes hydrolyzed stevioside and generated rubusoside, but substrate specificity analysis indicated that GH3-2 is the most suitable choice for the durative, steady, and high-efficiency production of rubusoside.

Key words: stevioside, rubusoside, bioconversion, response surface optimization, $\beta$-glucosidases

\section{Introduction}


Steviol glycosides (SGs) are natural low-calorie sweeteners found in the leaves ofStevia rebaudiana Bertoni, which has been approved as a food additive in many countries (Ahmad et al., 2020). They belong to a group of ent kaurene diterpene glycosides (Lopez-Carbon et al., 2019), that is, having the same backbone and differing in carbohydrate residues (Fig. 1a). SGs have more than 30 types, and the major components are stevioside (ST), rubusoside (RS), rebaudiosides A-F (RA-F), and dulcoside A (Table 1). The use and transport costs of SGs account for only $1 / 3$ and $1 / 200$ of sucrose, respectively, but its sweetness and calorific value are 300-450 times and 1/300 those of sucrose respectively. The lack of accumulation, toxicity, and carcinogenicity of SGs in human body (Zhao et al., 2020) make them highly recommended as natural substitutes of sucrose for individuals with diabetes or carbohydrate-controlled diets (Typek et al., 2020).

Steviosides are the most abundant component of SGs whereas are responsible for the characteristic bitter aftertaste (Bhardwaj et al., 2020; Chaturvedula \& Prakash, 2011; Hajihashemi \& Geuns, 2017). Comparatively, rubusoside (13-O-glucosyl-19-O-d-glucosyl-steviol) possesses more marvelous sweetness and flavorenhancing properties, earning itself praises as the most acceptable sweeteners. At present, rubusoside is mostly extracted and purified using chemical or mechanical methods from Rubus suavissimus, an endemic and rare shrub in Guangxi Province, directly leading to limited area of cultivation and climate fluctuation, along with economic and environmental burdens (Boonkaew et al., 2019).

Currently, food-grade SGs cost about 100 Yuan per $\mathrm{kg}$ at market rates, while extracts of $R$. suavissimus (rubusoside 20\%) cost 500-600 Yuan per kg. The price gap implies the great demand and expansive market prospects. In summary, innovation of the biotransformation methods of rubusoside would be advantageous for its large-scale production and broad application in the food and medical industries. The present study investigates the isolation, identification, and optimization of microorganisms directly transforming stevioside into rubusoside and analyzes the mechanism of enzymes in the transformation process to obtain the optimal enzyme.

\section{Materials and methods}

\section{Isolation and identification of endophytic bacterium}

Inorganic salt medium $\left(\mathrm{KH}_{2} \mathrm{PO}_{4} 3 \mathrm{~g} / \mathrm{L}, \mathrm{MgSO}_{4} 0.1 \mathrm{~g} / \mathrm{L}, \mathrm{K}_{2} \mathrm{HPO}_{4} 1.5 \mathrm{~g} / \mathrm{L}, \mathrm{CaCl}_{2} 0.01 \mathrm{~g} / \mathrm{L}\right.$, EDTA disodium $0.01 \mathrm{~g} / \mathrm{L}, \mathrm{pH}$ 7.5) containing only $10 \mathrm{~g} / \mathrm{L}$ stevioside was adopted as the selection medium to screen a mass of endophytic bacterium from S. rebaudiana Bertoni. Considering that the isolates were able to utilize steviosides and the release of D-glucose residue indicating the capacity of producing $\beta$-glucosidase, esculinMS medium was used for a second screening campaign in accordance with the method described by Xuan et al. with some modifications (Xuan, 2012). MS medium with double agar was supplemented with $0.2 \%$ esculin (6,7-2-hydroxy-coumarin- $\beta$-D-glucoside) and $0.6 \% \mathrm{FeCl}_{3}$ of the same volume. Observation of the wells surrounded by black or dark brown, indicating that esculin was hydrolyzed by $\beta$-glucosidase and underwent chromogenic reaction with $\mathrm{Fe}^{3+}$.

16S rDNA was amplified with the universal primer set 27F, 1492R and sequencing analysis was conducted by Sangon Biotech. A phylogenetic tree was constructed using the neighbor-joining method of the MEGA 6 program, and the bootstrap value was calculated based on 1000 replicates to obtain confidence levels for the branches. Scanning electron microscopy (SEM) of the bacterium was performed for species identification. The bacteria samples were observed by COXEM EM-30 (8.0nm @30KV SE, 20x-100,000x ) after pre-treatment including surface cleaning, fixation, rinsing and dehydration.

\subsection{Stevioside conversion experiment}

Isolates were precultured in Luria broth medium at $220 \mathrm{rpm}$ for $12 \mathrm{~h}$ at 37 , transferred into a fermentation medium (peptone $10 \mathrm{~g} / \mathrm{L}, \mathrm{KH}_{2} \mathrm{PO}_{4} 5 \mathrm{~g} / \mathrm{L}, \mathrm{K}_{2} \mathrm{HPO}_{4} 5 \mathrm{~g} / \mathrm{L}, \mathrm{NaCl} 2 \mathrm{~g} / \mathrm{L}, \mathrm{MgSO}_{4} 0.4 \mathrm{~g} / \mathrm{L}$, stevioside $10 \mathrm{~g} / \mathrm{L}$ ), and then incubated at $37,220 \mathrm{rpm}$ for 2 days. Then, $1 \mathrm{~mL}$ broth was collected every $24 \mathrm{~h}$ and centrifuged. Supernatants were preserved at -80 as test samples.

\subsection{Detection of rubusoside by thin-layer chromatography and}




\section{high-performance liquid chromatography}

Preliminary screening by TLC was performed with Silica gel G plates and butanol: acetic acid: diethyl ether: water (9:6:3:1, v/v/v/v) as its developing solvent (Teng et al., 2007). Standards of ST, RA, RS (10 $\mathrm{mg} / \mathrm{mL}$ ), and samples were sequentially pointed on the activated silica plate with capillary tubes. Spots on TLC plates were detected by spraying $10 \%(\mathrm{v} / \mathrm{v}) \mathrm{H}_{2} \mathrm{SO}_{4}$ in ethanol after adequately closed expansion and heated at $110{ }^{\circ} \mathrm{C}$ for $10 \mathrm{~min}$. Black spots appeared on the silicone plate in different positions as a result of carbonization.

After centrifugation and filtration, samples were reconfirmed using an HPLC system (Elite, Dalian, China) and a Agilent $\mathrm{HC}-\mathrm{C}_{18}$ column $(4.6 \times 150 \mathrm{~mm}$, ID $5 \mu \mathrm{m})$ with $\mathrm{NaH}_{2} \mathrm{PO}_{4}$ solution $(10 \mathrm{~g} / \mathrm{L})$ : acetonitrile $(32: 68$, $\mathrm{v} / \mathrm{v}$ ). The flow rate was $1 \mathrm{~mL} / \mathrm{min}$ and the detection level was UV $210 \mathrm{~nm}$ as described by $\mathrm{Li}$ et al with slight modifications (Li, 2010). Stevioside and rubusoside standards with different concentration gradients were also detected to draw standard curves.

\subsection{Experimental design for statistical analyses and optimization}

Medium with major inorganic salts means low yield and conversion efficiency. Response surface optimization was conducted to obtain the optimum medium composition and culture conditions. The impact of carbon sources (soluble starch, glucose, wheat bran, sucrose, lactose, corn pulp dry powder, $10 \mathrm{mg} / \mathrm{mL}$ ), nitrogen sources (peptone, beef extract, ammonium sulfate, fermented soybean meal, soy powder, yeast extract, 10 $\mathrm{mg} / \mathrm{mL}$ ), temperatures, and $\mathrm{pH}$ were preliminarily validated to reduce interference factors. Then, Min Run

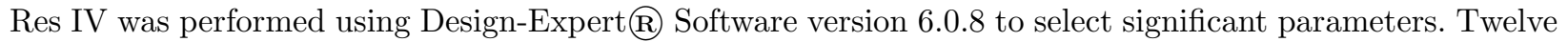
trial runs and two different levels were performed following the design as shown in Table S2. Manual mode and Main effect were used to analyze the interaction between variables on the results. Then design of the steepest ascent method was conducted (Table S4) followed by the above experimental results to enable the response value to quickly approach the maximum response center.

A Box-Behnken experiment was used to design a response surface analysis test (Table S5), the rubusoside yield was taken as the response value (Y), and these significant parameters were set as central points (Ko et al., 2012; Saikia et al., 2018). Experimental results were fitted to a second-order polynomial equation using multiple regression analysis. The accuracy of the design was expressed as the variation coefficient $(\mathrm{CV})$. The lower the CV value, the higher the reliability of the design.

\subsection{Complete genome sequence analysis}

Complete genome sequence analysis was performed by Biomarker Technologies to elucidate the mechanism of stevioside bioconversion. The whole genome sequence has been uploaded to NCBI (https://www.ncbi.nlm.nih.gov/) and the accession number is CP063663.

\subsection{Enzyme expression and purification}

According to the complete genome sequence of Lelliottia sp. accompanying with annotation and analysis based on Carbohydrate-Active enZYmes Database (CAZY), four possible genes named GH3-1 (2298 bp), GH3-2 (1428 bp), GH3-3 (2376 bp), and GH3-4 (3078 bp) were amplified with respective primers (Table S1) that introduced Hind III andEcoR I restriction sites. Amplification products with pet28a vectors were double-digested with restriction enzymes Hind III andEcoR $I$ and purified for ligation. The recombinants were transformed into Escherichia coli DH5 $\alpha$ strain. Subsequently, colonies on kanamycin media were verified and confirmed by colony PCR with sequencing primers (T7, T7-Ter) and plasmid DNA sequencing (Tatielle et al., 2018).

The recombinant plasmids were transformed into E. coli BL21, then for incubation with $0.5 \mathrm{mM}$ IPTG at 37 for $8 \mathrm{~h}$ (Udompaisarn et al., 2017). Bacterial cells were collected by centrifugation, and resuspended in $5 \mathrm{~mL}$ of Tris- $\mathrm{HCl}$ buffer with $1 \mathrm{mM}$ phenylmethanesulfonyl fluoride, then disrupted by sonication in an ice-water bath. The cell lysates were centrifuged and then purified using Ni-NTA His-binding Resin (7Sea Pharmatech Co., Ltd). 


\subsection{Enzyme assay}

\subsubsection{Effect of $\mathrm{pH}$ and temperature on enzyme activity}

$\beta$-glucosidase activity was carried out photometrically using $p$-nitrophenyl- $\beta$-D-glucopyranoside (pNPG) as a substrate. A $300 \mu \mathrm{L}$ mixture was composed of $260 \mu \mathrm{L}$ of PBS buffer, $30 \mu \mathrm{L}$ of $2 \mathrm{mM}$ pNPG, and $10 \mu \mathrm{L}$ of diluted enzyme. The reaction was stopped by adding $300 \mu \mathrm{L}$ of $1 \mathrm{M} \mathrm{NaCO}_{3}$ solution, and the amount of $p$-nitrophenol (pNP) was measured by the absorbance at $405 \mathrm{~nm}$ on a UV spectrophotometer (Dong et al., 2017). One unit of enzyme activity (U) was defined as the amount of enzyme that produced $1 \mu \mathrm{mol}$ of either pNP per minute.

The relative enzyme activity at different temperatures was compared varying from $25{ }^{\circ} \mathrm{C}$ to $65{ }^{\circ} \mathrm{C}$, and then the influence of $\mathrm{pH}$ on enzyme activity was investigated in the $\mathrm{pH}$ range of 3.0-10.0 with respective buffers. The result is expressed as the relative activity given the initial activity of 100\% (Ko et al., 2013; Wang et al., 2015).

\subsubsection{Effects of metal ions on enzyme activity}

The residual enzyme activities of the reaction systems with PBS buffer, enzyme, and metal ions ( $5 \mathrm{mM} / \mathrm{L})$ were measured under optimal conditions, whereas those of others without metal ions served as the control group (Ikezawa et al., 1986).

\subsection{Substrate specificity}

The assay of substrate specificity was performed by determining the enzyme activity in buffers containing 2 $\mathrm{mM}$ gentiobiose, trehalose, lactose, sucrose, maltose, salicin, chondroitin disaccharide, melibiose, and amygdalin at $50{ }^{\circ} \mathrm{C}$ for $20 \mathrm{~min}$. ST, RS, RA, RD, and RM were also selected as substrates to explore the mutual transformation of stevia glycosides (Boonkaew et al., 2019; Hu et al., 2012).

\section{Results and Discussion}

\subsection{Screening and identification of transformed bacterium}

A total of 46 isolates were preliminarily screened and purified using steviosides as the sole carbon source. In microorganisms, $\beta$-glucosidases ( $\beta$-D-glucoside glucohydrolase, E.C. 3.2.1.21) are mainly involved in glucose metabolism in vivo. They catalyze the hydrolysis of $\beta$-glucosidic linkages of various oligosaccharides and aryl-glycosides, thus releasing the sugar moiety and the aglycone (Yoshiara et al., 2018). Esculin generates glucose and esculetin (6, 7-2-hydroxy-coumarin) under the action of $\beta$-glucosidase, and the interaction of esculetin with $\mathrm{Fe}^{3}+$ appears black or dark brown. $\beta$-glucosidases come from a wide range of sources and can be detected almost in any carbohydrate-based organism with a cellular structure. Therefore, esculin-MS medium was used to screen isolates producing $\beta$-glucosidases, and 34 isolates appeared black or dark brown (Fig. 1b).

\subsection{Thin-layer chromatography and high-performance liquid chromatography detection}

Given the wide range of activity of $\beta$-glucosidases, fermentation liquids of isolates were detected by TLC preliminarily. This method is reliable to some extent, considering the distinct differences among various standards (Fig. 1c). Finally, HPLC was performed for reconfirmation. Fig.1d show the disappearance of ST and occurrence of rubusoside without obvious byproducts, indicating that LST-1 was the target strain that specifically converts stevioside into rubusoside. Despite the temporary low conversion rate $(12 \%-17 \%)$ due to the inappropriate culture medium, the unique specific transformation of LST-1 makes its hidden potential worth unlocking.

Standard curves were obtained according to the peak area with different concentrations of stevioside and rubusoside, where $\mathrm{Y}$ represents the peak area and $\mathrm{x}$ represents the concentration. The functions were used to calculate the conversion rate.

\subsection{Species identification and complete genome sequence analysis}


Results of $16 \mathrm{~S}$ rDNA sequence analysis indicated that LST-1 shared high sequence similarities to Lelliottia jeotgali (99.85\%), Lelliottia nimipressuralis (98.82\%), Lelliottia amnigena(98.54\%). Morphological identification suggested that LST-1 is Gram-negative, atrichous, short rod-shaped bacteria (Fig. 2d). Combined with comparative phylogenetic tree analysis based on 16S rDNA gene sequences and multilocus sequence typing (MLST) (Fig.2e,2f), LST-1 was classified as Lelliottia genus.

The genome of Lelliottia sp. LST-1 is composed of a chromosome with 4,611,055 base pairs (Fig.2a) and a plasmid with 39,319 base pairs (Fig.2b). The outermost circle represents genome size, and each scale is 5 KB. The second and third circles are genes on the plus and minus chains of the genome, respectively, and different colors represent different functional classifications of Cluster of Orthologous Groups (COG). The fourth circle is the repetition sequence. The fifth circle represents tRNA (blue) and rRNA (purple). GC content is displayed on the sixth circle. The light yellow part indicates that the GC content in this region is higher than the average GC content of the genome, while the blue part indicates that the GC content in this region is lower than the average GC content of the genome. The total length of the predicted coding gene sequence is 4,140,156. Gene function annotation analysis was conducted after Blast with functional databases, such as COG, Kyoto Encyclopedia of Genes and Genomes, Swiss-Prot, Non-Redundant Protein Database.

\subsection{Optimization ofrubusoside production}

The effects of diverse carbon sources, nitrogen sources, temperatures, and $\mathrm{pH}$ were examined to enlarge rubusoside production. Simple sugars readily hydrolyzed to glucose are not conducive to the conversion because of glucose inhibition effect (Pang et al., 2017), whereas organic nitrogen sources favor the conversion process because of their abundant nutrients. As shown in Fig. 3, LST-1 largely accumulated rubusoside in the presence of wheat bran, corn pulp dry powder, fermented soybean meal, and soy powder under the conditions of 37 and $\mathrm{pH}$ 7.0. This result is consistent with common theory.

These particular carbon and nitrogen sources were selected to perform Min Run Res IV. Multiple regression equation obtained is $\mathrm{R} 1=+2833.96+532.71 \mathrm{~A}+297.58 \mathrm{~B}+23.02 \mathrm{C}+391.28 \mathrm{D}-69.00 \mathrm{E}$. Analysis of variance results of the Min Run Res IV model fitting are presented in Table S3. The determination coefficient $\left(\mathrm{R}^{2}\right)$ was 0.9683 , indicating that the model does not explain only $3.17 \%$ of the total variations. The Model Fvalue of 27.73 implies the model is significant. Wheat bran, fermented soybean meal, and corn pulp dry powder efficiently promoted the bioconversion of stevioside to rubusoside. Subsequent design of the steepest ascent method approximated significant factors to the optimal response region. Apparently, the highest yield condition was $1.1 \%$ wheat bran, $0.5 \%$ fermented soybean meal, and $0.7 \%$ corn pulp dry powder (Table S4). Thus, it was taken as the central point of subsequent tests with some modifications.

A Box-Behnken experiment was used to design a response surface analysis with three factors and three levels, and $1 \%$ wheat bran, $0.5 \%$ fermented soybean meal, and $0.7 \%$ corn pulp dry powder were set as the central point. The experimental results were fitted with a quadratic polynomial model by using designExpert 12.0, and its mathematical model was $\mathrm{R} 1=+4481.08+396.11 \mathrm{~A}+65.86 \mathrm{~B}+45.07 \mathrm{C}-63.51 \mathrm{AB}-101.89 \mathrm{~A}$ $\mathrm{C}+222.72 \mathrm{BC}-603.94 \mathrm{~A}^{2}-395.03 \mathrm{~B}^{2}-291.54 \mathrm{C}^{2}$.

Variance analysis results of Box-Behnken model fitting are presented in Table S6. A relatively lower value of CV (1.34\%) indicates better precision and reliability of experiments. The Model F-value of 184.11 implies the model is significant, the predicted $\mathrm{R}^{2}$ of 0.9402 is in reasonable agreement with the adjusted $\mathrm{R}^{2}$ of 0.9904 , and the difference is less than 0.2 , indicating the good reliability and fitness of the model. The 3D response surface plots (Fig. 6a) show that the interaction between each independent variable is good and that the best prediction point is within the scope of experimental investigation. The maximum predicted response was $5.012 \mathrm{mg} / \mathrm{mL}$, followed by wheat bran $(1.374 \%)$, fermented soybean meal (0.337\%), and corn pulp dry powder $(0.464 \%)$.

In the current research, the conversion of stevioside is defined as the consumption rate of stevioside, and the output rate of rubusoside is defined as the rate of rubusoside production with stevioside consumption. Purely from the conversion rate, LST-1 completely utilized stevioside, as evidenced by its absence in the 
fermentation broth by HPLC within $24 \mathrm{~h}$. The output rate was approximately $75.4 \%$, illustrating the high efficiency, utilization level, and specificity of the substrate. Compared with the initial conversion rate $(8 \%-$ $12 \%$ ), the optimized conversion rate increased up to six to nine times. Few publications described rubusoside biotransformation by bacterium. Jiang et al. identified a Chryseobacterium species that converts stevioside into rubusoside by intracellular enzyme, obtaining $5.7 \mathrm{~g} / \mathrm{L}$ rubusodide solution after $48 \mathrm{~h}$ with $10 \mathrm{~g} / \mathrm{L}$ stevia glycosides, including $7.2 \mathrm{~g} / \mathrm{L}$ stevioside (Jiang et al., 2011). Nevertheless, LST-1 has a distinct advantage because of its high-to-excellent yields and short reaction times.

\subsection{Enzyme purification and properties}

Approximately $1 \mathrm{~mL}$ of enzymes were obtained after centrifugation through an ultrafiltration device for desalinization. The molecular masses of the purified enzymes on SDS-PAGE were about 85, 55, 86, and 116 $\mathrm{kDa}$, respectively (Fig.4), which is consistent with the calculated molecular mass of the predicted amino acid sequence. The concentrations of the purified enzymes were $129.77,225.53,119.15$, and $172.34 \mu \mathrm{g} / \mathrm{mL}$.

The enzymatic activities of the recombinants were measured at various temperatures $(30-70)$ using pNPG as the substrate. Results showed that the activity increased within the temperature range of $30{ }^{\circ} \mathrm{C}-50{ }^{\circ} \mathrm{C}$ and gradually decreased as the temperature was increased; the optimal temperature of the recombinants was approximately 50 (Fig. 5a). This result is roughly coincident with the optimal temperature of known $\beta$-glucosidases at $40-110$ (Pan \& Luo, 2006). In general, four enzymes retained over $80 \%$ of their activity for $3 \mathrm{~h}$ at 40 and exhibited poor thermostability at $60{ }^{\circ} \mathrm{C}$ (Fig. 5e, 5f, 5g, 5h). By comparison, GH3-1 and GH3-2 stabilized weekly in excessive temperatures than the other two. In general, the optimum temperatures of mesophilic $\beta$-glucosidases range from $30{ }^{\circ} \mathrm{C}$ to $65{ }^{\circ} \mathrm{C}$, and inactivation mostly occurs at $55{ }^{\circ} \mathrm{C}$ to $70{ }^{\circ} \mathrm{C}$ (Dharmawardhana et al., 1995; Nagano et al., 2005)

The enzymatic activities of recombinants were measured at various $\mathrm{pH}$ values $(\mathrm{pH} 2-10.0)$. The optimum $\mathrm{pH}$ value of the four enzymes was 6 and displayed high activity (over 80\%) in weak acid to neutral conditions (Fig.5c). The enzymes also showed good and extensive $\mathrm{pH}$ stability, retaining over $80 \%$ of its original activity between $\mathrm{pH} 4$ and 8 (Fig. 5d). However, under strong acid and strong base conditions, the enzyme activity and stability obviously reduced and declined. As reported in the literature, most $\beta$-glucosidases exhibit good acid and alkaline resistance, scilicet good viability and stability in a wide range of $\mathrm{pH}$ (Yao et al., 2018).

Metal ions $(5 \mathrm{mmol} / \mathrm{L})$ displayed different influences on the four enzymes. SDS, $\mathrm{Cu}^{2+}$, and $\mathrm{Co}^{2+}$ remarkably inhibited the activity of the enzymes. This result may be ascribed to the fact that heavy metal ions inactivate enzymes by binding or reacting with their related groups or destroy some of the forces that stabilize the enzyme molecules (Hu et al., 2016). $\mathrm{Mn}^{2+}, \mathrm{Ca}^{2+}$, and $\mathrm{Zn}^{2+}$ exerted minimal effect, whereas $\mathrm{Fe}^{2+}$ and $\mathrm{Mg}^{2+}$ significantly promoted their activity up to 1.75 times (Fig. 5b). The reaction solution shows that proteins formed complexes with metal ions, thus accelerating the reaction. However, the effects of metal ions on different enzymes varied greatly because of the differences in their spatial structure and composition.

\subsection{Assay of substrate specificity}

The substrate specificity of the four enzymes with different glycosides and saccharides were determined. As shown in Table 2 , the enzymes showed the broad substrate hydrolysis of $\alpha(\alpha 1-1, \alpha 1-4, \alpha 1-6)$ - and $\beta$ ( $\beta 1-2, \beta 1$ $4, \beta 1-6)$-glycosidic bonds. The rare catalytic activities of rebaudioside $\mathrm{A}$, rebaudioside $\mathrm{D}$, and rebaudioside $\mathrm{M}$ can be ascribed to the steric hindrance of a $\beta$-glucosyl (1-3) derivative of the $\mathrm{C}_{13}$-hydroxyl group (Wang et al., 2015), which fit to most reported steviosides hydrolyzing $\beta$-glucosidases. Meanwhile, no hydrolytic activity was detected for amygdalin and salicin compared with other $\beta$-glucosidases hydrolyzing stevioside (Boonkaew et al., 2019; Qing et al., 2019; Wang et al., 2015), signifying higher specificity to glycosides than reported. Relatively weak activity for melibiose and specific hydrolysis of trehalose by GH3-3 were also observed. In sum, high specificity for glycosides and broad hydrolysis of saccharides make the four enzymes competitive in actual production.

All enzymes hydrolyzed stevioside and generated rubusoside (Fig.6b). The substrate stevioside can be completely consumed within $2 \mathrm{~h}$, and the yields of rubusoside were $2.49 \pm 0.19,2.40 \pm 0.11,2.96 \pm 0.07$, and 
$1.49 \pm 0.036 \mathrm{mg} / \mathrm{mL}$. In theory, the presumable yield of rubusoside was $3.32 \mathrm{mg} / \mathrm{mL}$, and the output rates were respectively $75 \%, 72.29 \%, 89.16 \%$, and $44.88 \%$. GH3-3 had the highest output rate, but only GH3-2 displayed no activity for rubusoside, implying specific cleavage of glycosidic bond and glucose residue.

Only a few $\beta$-glucosidases convert stevioside to rubusoside, including S-producing $\beta$-glucosidase (SPGase), SPBGL1, and BGL1 (Jin-A Ko et al., Qing Lan et al., Zilong Wang et al.). As shown in Table 3, GH3-2 displayed higher output rate $(72.29 \%)$ with $23.83 \mu \mathrm{g} / \mathrm{mL}$ enzyme than SPGase $(64 \%, 33.2 \mu \mathrm{g} / \mathrm{mL})$, BGL1 $(78.8 \%, 55 \mu \mathrm{g} / \mathrm{mL})$. SPBGL1 $(99 \%, 600 \mu \mathrm{g} / \mathrm{mL})$ had the highest conversion rate, but the substrate concentration and enzyme amount considerably increased with the longest reaction time in reverse. SPBGL1 showed high activity $(>80 \%)$ in very narrow ranges (pH5.0-6.5) and greatly restricted the large-scale fermentation production. In addition, GH3-2 could improve the output rate by adjusting the ratio of substrate concentration to enzyme. The above analysis indicates that GH3-3 is a promising candidate in industrial production.

\section{Declaration of Competing Interest}

The authors declare that they have no known competing financial interests or personal relationships that could have appeared to influence the work reported in this paper.

\section{Acknowledgments}

This research was supported by the Natural Science Foundation of Zhejiang Province (19042049-D) and National Innovation and Entrepreneurship Training Program for College Students (202010338038).

\section{Reference}

Ahmad, J., Khan, I., Blundell, R., Azzopardi, J., \& Mahomoodally, M.F. (2020).Stevia rebaudiana Bertoni $\therefore$ an updated review of its health benefits, industrial applications and safety. Trends in Food Science and Technology , 100, 177-189. doi:10.1016/j.tifs.2020.04.030

Bhardwaj, V., Singh, R., Singh, P., Purohit, R., \& Kumar, S. (2020). Elimination of bitter-off taste of stevioside through structure modification and computational interventions. Journal of Theoretical Biolog y, 486, 110094. doi:10.1016/j.jtbi.2019.110094

Boonkaew, B., Udompaisarn, S., Arthan, D., \& Somana, J. (2019). Expression and characterization of a recombinant stevioside hydrolyzing beta-glycosidase from Enterococcus casseliflavus . Protein Expression and Purification , 163, 105449. doi:10.1016/j.pep.2019.105449

Chaturvedula, V.S.P., \& Prakash, I. (2011). Structures of the novel diterpene glycosides from Stevia rebaudiana . Carbohydrate Research , 346, 1057-1060. doi:10.1016/j.carres.2011.03.025

Dharmawardhana, D.P., Ellis, B.E., \& Carlson, J.E. (1995). A beta-glucosidase from lodgepole pine xylem specific for the lignin precursor coniferin. Plant physiology , 107, 331-339. doi:10.1104/pp.107.2.331

Dong, W., Xue, M., Zhang, Y., Xin, F., Wei, C., Zhang, W., Wu, H., Ma, J., \& Jiang, M. (2017). Characterization of a $\beta$-glucosidase fromPaenibacillus species and its application for succinic acid production from sugarcane bagasse hydrolysate. Bioresource Technology , 241, 309-316. doi:10.1016/j.biortech.2017.05.141

Hajihashemi, S., \& Geuns, J.M.C. (2017). Steviol glycosides correlation to genes transcription revealed in gibberellin and paclobutrazol-treatedStevia rebaudiana. Journal of Plant Biochemistry and Biotechnology , 26, 387-394. doi:10.1007/s13562-017-0399-5

Hu, D., Liu, C., \& Liu, S. (2016). The Effects of Different Kinds of Salts on the $\beta$-Glycosidase Enzymes in Rubber Seed. The Food Industry, 37, 71-73.

Hu, K.L., Han, J., Liu, W.F, Wang, Y.P, \& Tao, Y. (2012). Cloning, expression and characterization of a beta-glucosidase from Geobacillus thermodenitrificans. Microbiology China , 39, 891-900. 
Ikezawa, H., Matsushita, M., Tomita, M., \& Taguchi, R. (1986). Effects of metal ions on sphingomyelinase activity of Bacillus cereus. Archives of Biochemistry and Biophysics , 249, 588-595. doi:10.1016/00039861(86)90037-8

Jiang, Z., Chen, Y., \& Liu, H. (2011). Identification and biotransformation properties of a bacterium that converts stevioside into rubusoside. Acta microbiology Sinca , 51, 43-49.

Ko, J.A., Kim, Y.M., Ryu, Y.B., Jeong, H.J., Park, T.S., Park, S.J., Wee, Y.J., Kim, J.S., Kim, D., \& Lee, W.S. (2012). Mass Production of Rubusoside Using a Novel Stevioside-Specific beta-Glucosidase fromAspergillus aculeatus . Journal of Argiculture Food Chemistry , 60, 6210-6216. doi:10.1021/jf300531e

Ko, J.A., Ryu, Y.B., Kwon, H.J., Jeong H.J., Park S.J., Kim C.Y., Wee, Y.J., Kim, D., Lee W.S., \& Kim, Y,M. (2013). Characterization of a novel steviol-producing $\beta$-glucosidase from Penicillium decumbensand optimal production of the steviol. Applied Microbiology and Biotechnology , 97, 8151-8161. doi:10.1007/s00253013-4883-0

Li, R. (2010). Determination of steviol glycosides in food by high performance liquid chromatography. Journal of Anshan Normal University, 12, 44-45.

Lopez-Carbon, V., Sayago, A., Gonzalez-Dominguez, R., \& Fernandez-Recamales, A. (2019). Simple and Efficient Green Extraction of Steviol Glycosides from Stevia rebaudiana Leaves. Foods . 8, 402-412. doi:10.3390/foods 8090402

Nagano, A.J., Matsushima, R., \& Nishimura, I.H. (2005). Activation of an ER-body-localized $\beta$-Glucosidase via a Cytosolic Binding Partner in Damaged Tissues of Arabidopsis thaliana . Plant and Cell Physiology , 46, 1140-11488. doi:10.1093/pcp/pci126

Pan, L.H., \& Luo, J.P. (2006). Advance in Research and Application of $\beta$-D-glucosidase. Food Science , 27, 803-807.

Pang, P.J., Cao, L.C., Liu, Y.H., Xie, W., \& Wang, Z., 2017. Structures of a glucose-tolerant $\beta$-glucosidase provide insights into its mechanism. Food Science, 198, 154-162.

Lan, Q., Tang, T.T.,Yin, Y., Qu, X.Y., Wang, Z.L., Pang, H., Huang, R.B., \& Du, L.Q. (2019). Highly specific sophorose $\beta$-glucosidase fromSphingomonas elodea ATCC 31461 for the efficient conversion of stevioside to rubusoside. Food Chemistry , 295, 563-568. doi:10.1016/j.foodchem.2019.05.164

Saikia, R., Baruah, B., Kalita, D., Pant, K.K., Gogoid, N., \& Katakia, R. (2018). Pyrolysis and kinetic analyses of a perennial grass (Saccharum ravannae L.) from north-east India: Optimization through response surface methodology and product characterization.Bioresource Technology , 253, 304-314. doi:10.1016/j.biortech.2018.01.054

Silva, T.P., de Albuquerque, F.S., Santos, C.W.V.D., Franco, M., Caetano, L.C., \& Pereira, H.J.V. (2018). Production, purification, characterization and application of a new halotolerant and thermostable endoglucanase of Botrytis ricini URM 5627. Bioresource Technology , 270, 263-269. doi:10.1016/j.biortech.2018.09.022

Teng, X.J., Yang, D., Meng, T., \& Hao, Z.B. (2007). Thin layer chromatography analysis of steviol glycosides. Sugar Crops of China . 04, 24-25,31.

Typek, R., Dawidowicz, A.L., \& Stankevic, M. (2020). Stability of stevioside in food processing conditions: unexpected recombination of stevioside hydrolysis products in ESI source. Food Chemistry, 331, 127262. doi:10.1016/j.foodchem.2020.127262

Udompaisarn, S., Arthan, D., \& Somana, J. (2017). Development and Validation of an Enzymatic Method To Determine Stevioside Content from Stevia rebaudiana. Journal of Agricultural and Food Chemistry , 65, 3223-3229. doi:10.1021/acs.jafc.6b05793 
Wang, Z.L., Wang, J.P, Jiang, M.H., Wei, Y.T., Pang, H., Wei, H., Huang, R.B., \& Du, L.Q. (2015). Selective production of rubusoside from stevioside by using the sophorose activity of $\beta$-glucosidase fromStreptomyces sp. GXT6. Applied Microbiology and Biotechnology , 99, 9663-9674. doi:10.1007/s00253-015-6802-z

Xuan, Y. (2012). Isolation and purification of stevia glycoside invertase and microbial transformation of steviol, Nanjing Normal University.

Yao, Y., Liu, Q., Liu, F., Yao, Y.T., Zhu, S.L., \& Jiang, H.Y. (2018). Characteristics of $\beta$-glucosidase and its Applications in Food Processing. Guizhou Agricultural Science, 46, 132-135.

Yoshiara, L.Y., Madeira, T.B., de Camargo, A.C., Shahidi, F., \& Ida, E.I. (2018). Multistep Optimization of $\beta$-Glucosidase Extraction from Germinated Soybeans (Glycine max L. Merril) and Recovery of Isoflavone Aglycones.Foods , 7 , 110-123. doi:10.3390/foods7070110

Zhao, R.R., Wang, J.P., Qin, L.H., Zhang, X., \& Mei, Y.W. (2020). Stevioside improved hyperglycemiainduced cardiac dysfunction by attenuating the development of fibrosis and promoting the degradation of established fibrosis. Journal of Functional Foods , 68, 103895. doi:10.1016/j.jff.2020.103895

\section{List of Tables}

Table 1 Structure and characteristics of steviol glycosides

\begin{tabular}{llllll}
\hline Name & $\mathrm{R}_{1}\left(\mathrm{C}_{19}\right)$ & $\mathrm{R}_{2}\left(\mathrm{C}_{13}\right)$ & Molecular weight & Sweetness & Proportion of total glycosides \\
\hline Stevioside & Glc- & Glc-2Glc- & 804.88 & $100-300$ & $50 \%-70 \%$ \\
Rebaudioside A & Glc- & Glc-3Glc-Glc- & 967.01 & $300-400$ & $15 \%-20 \%$ \\
Rebaudioside B & H- & Glc-3Glc-2Glc & 804.88 & $10-15$ & less \\
Rebaudioside C & Glc- & Glc-3Glc-2Rha & 951.03 & $40-60$ & $10 \%-15 \%$ \\
Rebaudioside D & Glc-2Glc & Glc-3Glc-Glc- & 1129.15 & $200-250$ & less \\
Rebaudioside E & Glc-2Glc & 2Glc-Glc- & 967.01 & $100-150$ & less \\
Rebaudioside G & Glc- & Glc-2Glc- & 804.88 & Not clear & less \\
Rebaudioside M & Glc-2Glc -3Glc & Glc-2Glc-3Glc & 1291.29 & $160-500$ & less \\
Durcoside A & Glc- & Glc-2Rha- & 788.88 & $40-60$ & less \\
Steviolbioside & H- & Glc-2Glc- & 642.74 & $10-15$ & less \\
Rubusoside & Glc- & Glc- & 642.74 & {$[?] 300$} & less \\
\hline
\end{tabular}

Table 2 Substrate specificity of four enzymes

\begin{tabular}{llllll}
\hline Substrate & Linkage of glycosyl group & GH3-1 & GH3-2 & GH3-3 & GH3-4 \\
\hline ST & Glc $\beta$-(1-2) Glc & 100 & 100 & 100 & 100 \\
RA & Glc $\beta$-(1-2)/Glc $\beta$-(1-3) Glc & 0 & 0 & 0 & 0 \\
RD & Glc $\beta$-(1-2)/Glc $\beta$-(1-3) Glc & 0 & 0 & 0 & 0 \\
RM & Glc $\beta$-(1-2)/Glc $\beta$-(1-3) Glc & 0 & 0 & 0 & 0 \\
RS & $\beta$-Glc & $24.78 \% \pm 0.53$ & 0 & $40.71 \% \pm 0.23$ & $37.21 \% \pm 0.58$ \\
Amygdalin & $\beta$-Glc & 0 & 0 & 0 & 0 \\
Salicin & $\beta$-Glc & 0 & 0 & 0 & 0 \\
Lactose & Gal $\beta$-(1-4) Glc & $42.59 \% \pm 0.17$ & $64.96 \% \pm 0.08$ & $49.80 \% \pm 0.29$ & $200.87 \% \pm 0.44$ \\
Sucrose & Glc $\alpha-(1-2)$ Fru & 0 & 0 & 0 & 0 \\
Gentiobiose & Glc $\beta-(1-6)$ Glc & $61.11 \% \pm 0.41$ & $69.81 \% \pm 0.40$ & $48.98 \% \pm 0.30$ & $133.91 \% \pm 0.19$ \\
Trehalose & Glc $\alpha-(1-1)$ Glc & 0 & 0 & $36.68 \% \pm 0.41$ & 0 \\
Maltose & Glc $\alpha-(1-4)$ Glc & $58.20 \% \pm 0.20$ & $48.72 \% \pm 0.34$ & $46.61 \% \pm 0.42$ & $207.83 \% \pm 0.22$ \\
melibiose & Glc $\alpha-(1-6)$ Gal & $22.22 \% \pm 0.35$ & $49.29 \% \pm 0.58$ & 0 & $50.43 \% \pm 0.15$ \\
Chondroitin & Glc $\beta-(1-3)$ Glc & 0 & 0 & 0 & 0 \\
\hline
\end{tabular}


*Activities on glycosides and saccharides were determined as relative values of the percentage activity obtained with stevioside.

Table 3 Enzymatic ability of $\beta$-glucosidases on stevioside.

\begin{tabular}{llllll}
\hline Enzyme & Substrate & The amount of enzyme & Reaction Time & Conversion rate & Yield rate \\
\hline SPGL1 & $24 \%$ ST (W/V) & $600 \mu \mathrm{g} / \mathrm{mL}$ & $6 \mathrm{~h}$ & $98.6 \%$ & $99 \%$ \\
BGL1 & $1 \% \mathrm{ST}(\mathrm{W} / \mathrm{V})$ & $30 \mu \mathrm{mL}$ & $6 \mathrm{~h}$ & $98.2 \%$ & $78.8 \%$ \\
GH3-2 & $1 \% \mathrm{ST}(\mathrm{W} / \mathrm{V})$ & $23.83 \mu \mathrm{g} / \mathrm{mL}$ & $2 \mathrm{~h}$ & $100 \%$ & $72.29 \%$ \\
\hline
\end{tabular}

\section{List of figure legends}

Figure .1a General formula of steviol glycosides. R1 represents substituents in $\mathrm{C}_{19}$, R2 represents substituents in $\mathrm{C}_{13}$.

Figure .1b Color reactions of esculin hydrolysis. CK: distilled water. LST-1:100 $\mu$ L fermentation broth. Esculetin interacting with $\mathrm{Fe}^{3+}$ appears black or dark brown.

Figure .1c TLC detection of steviol glycoside. Spots of rebaudioside A, stevioside, and rubusoside are standards $(10 \mathrm{mg} / \mathrm{mL}), \mathrm{LST}-1$ is fermented broth, and negative control is the fermentation medium.

Figure .1d HPLC profiles of the hydrolysis product from LST-1. Dotted lines depict standards of ST and RS, and solid lines represent consumption of ST and production of RS.

Figure. 2a,b Representation of the LST-1 chromosome. Predicted coding regions on the strand were classified as functions according to color

Figure. 2c Results of CAZy annotation. The database consists of the following enzyme families: glycoside hydrolases (GHs), glycosyl transferases (GTs), polysaccharide lyases (PLs), carbohydrate esterases (CEs), auxiliary activities (AAs), carbohydrate-binding modules (CBMs).

Figure. 2d Scanning electron microscope observation

Figure. 2e, 2f Phylogenetic tree of Lelliottia andEnterobacter strains based on 16S rDNA sequences by the maximum-likelihood method (e). MLST analysis using concatenated partial recA, atpD, and infB gene sequences $(\mathrm{f})$.

Figure. 3 Optimization of different components of medium and various culture conditions. (a) Different carbon sources. (b) Different nitrogen sources. (c) Optimization of pH. (d) Optimization of temperature.

Figure. 4 SDS-PAGE analysis of recombinants. M: molecular mass marker. 1: vector control; 2: crude enzyme of GH3-1; 3: crude enzyme of GH3-2; 4: crude enzyme of GH3-3; 5: crude enzyme of GH3-4.

Figure. 5a, 5b, 5c, 5d Effect of temperature and $\mathrm{pH}$ on enzyme activity. Results are expressed as the relative activity given the initial activity of 100\%. (a) Temperature optimum, (b) Effects of metal ion, (c) $\mathrm{pH}$ optimum, and (d) pH stability.

Figure. 5e, 5f, 5g, 5h Thermal stability of GH3-1, GH3-2, GH3-3, GH3-4 respectively. Enzymes were incubated at $40{ }^{\circ} \mathrm{C}$ to $60{ }^{\circ} \mathrm{C}$, and activities were detected at $\mathrm{pH} 6.0$ and $50{ }^{\circ} \mathrm{C}$.

Figure. 6a Response surface plot of A (wheat bran), B (fermented soybean meal), C (corn pulp dry powder) on responses $(\mathrm{R} 2)$

Figure. 7b HPLC analysis of stevioside hydrolysis. a: Standard stevioside, rubusoside. b: Stevioside hydrolysate by GH3-1. c: Stevioside hydrolysate by GH3-2. d: Stevioside hydrolysate by GH3-3. e: Stevioside hydrolysate by GH3-4.

\section{Hosted file}


Figure.pdf available at https://authorea.com/users/376517/articles/493472-directionalbioconversion-and-optimization-of-stevioside-into-rubusoside-by-lelliottia-sp-lst-1 